commercial chemical synthesis that involves around 50 steps. In addition, the two new syntheses have yields of $45 \%$ and $37 \%$, respectively - a considerable improvement on current synthetic methods.

The authors say that their method could one day generate heparin efficiently and on an industrial scale. Science 334, 498-501 (2011)

\section{CARDIOVASCULAR BIOLOGY}

\section{Fats boost python hearts after meal}

The Burmese python's heart increases in mass by an astounding $40 \%$ during the two to three days that follow a large meal, thanks in part to a specific set of fat molecules in the blood.

This enlargement helps the python to digest huge feasts. Leslie Leinwand at the University of Colorado in Boulder and her colleagues examined hearts in this snake species, Python molurus (pictured), before and after a meal. Instead of seeing signs of cell division in the postprandial heart, they found that the heart cells had expanded. This was accompanied by activation of fatty-acid transport and oxidation, and by greater expression and activity of superoxide dismutase - a cardioprotective, free-radicalscavenging enzyme - in the heart.

The authors also identified a combination of fatty acids in postprandial python blood

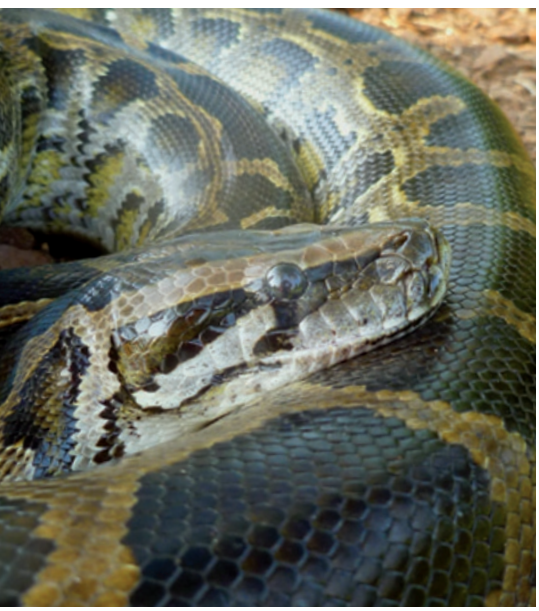

that promoted healthy cardiac growth when injected into both pythons and mice. This might offer a therapeutic approach for diseased human hearts.

Science 334, 528-531 (2011)

\section{IMMUNOLOGY \\ Immune system's bacterial shepherd}

Platelets have roles in blood clotting and inflammation, and may also be required for the interaction between the 'sentinel' cells of the immune system and certain bloodborne bacteria.

Admar Verschoor and Dirk Busch at the Technical University of Munich in Germany and their colleagues monitored infection by the bacterium Listeria monocytogenes in mice. They found that platelets clustered around the bacteria, shuttling them to the spleen, where immune cells called dendritic cells engulf the pathogen. These are the first steps to mounting an immune response involving cell-killing T cells. The authors identified a key immune-system protein and a platelet receptor that are necessary for the association of platelets with L. monocytogenes, as well as with other clinically relevant bacteria.

Nature Immunol. http://dx.doi. org/10.1038/ni.2140 (2011)

\section{PUBLIC-HEALTH ECOLOGY}

\section{Sterile males for mosquito control}

A field trial in the Cayman Islands has shown that male mosquitoes engineered to produce non-viable offspring can compete with normal males for mates. This is a key step towards controlling populations of the diseasecarrying insect.

Mosquito control is the only available strategy for tackling dengue, a viral disease spread mainly by the Aedes aegypti mosquito for which there is no vaccine. Luke Alphey at Oxitec in Oxford, UK, and his colleagues tested males of an

COMMUNITY CHOICE

The most viewed papers in science

NEUROSCIENCE

\title{
Vertigo in the MRI machine
}

\section{HIGHLY READ \\ on www.cell.com 25 Sept-25 Oct}

the fluid in the inner ear

The vertigo that many people experience when near or inside magnetic resonance imaging (MRI) machines may be due to a direct effect of the strong magnetic field on

The labyrinth of the inner ear controls balance and consists of semicircular canals filled with ionic fluid. Dale Roberts at Johns Hopkins University in Baltimore, Maryland, and his colleagues placed ten healthy volunteers and two patients with dysfunctional labyrinths into MRI scanners, and exposed them to a static magnetic field. The healthy people - but not those with damaged labyrinths - continuously flicked their eyes sideways, as if to correct for perceived head movements. The pattern of eye movement depended on the polarity of the magnetic field and the orientation of the head.

The authors say that their data can best be explained by the action of a particular magnetic force, the Lorentz force, on the potassium-ion-rich labyrinth fluid. This force pushes the fluid against pressure sensors to bring about the sensation of vertigo. Curr. Biol. 21, 1635-1640 (2011)

engineered strain of A. aegypti, which produce offspring that don't survive into adulthood and also express a fluorescent marker. The researchers released the mosquitoes in a 10-hectare trial area over a four-week period, so that the engineered insects made up about $16 \%$ of the adult male population. Of larvae caught in traps, 9.6\% were fluorescent, indicating successful mating by the engineered males.

The authors estimate that the release rate would need to be between 1.4 - and 12 -fold that used in this trial to suppress the natural mosquito population. Nature Biotechnol. http://dx.doi. org/10.1038/nbt.2019 (2011)

When sand
dunes collide

Sand dunes can appear to pass through each other when they collide, confirming previous theoretical predictions.

Pieter Vermeesch at the University of London studied 45 years' worth of satellite images of a type of

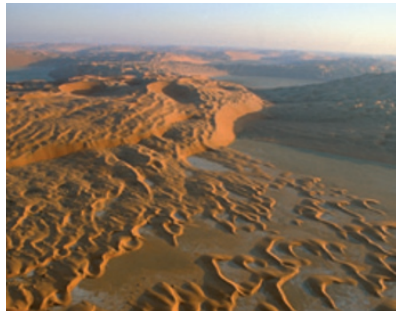

dune in Chad called barchan (pictured), and observed ten such collisions.

When wind pushes a smaller dune towards a larger, slower-moving one, the small one catches up with the larger dune. Once there, the smaller dune blocks the supply of sand to the larger one such that the small one grows and slows, and the large one shrinks and accelerates. The transfer of mass means that one dune appears to move through the other as if it were a solitary wave.

Geophys Res. Lett. http://dx.doi. org/10.1029/2011GL049610 (2011)

\section{$\rightarrow$ NATURE.COM}

For the latest research published by Naturevisit:

www.nature,com/latestresearch 